Opt. Commun. 254 (2005) 353-360

\title{
Amphoteric refraction at the interface between isotropic and anisotropic media
}

\author{
Hailu Luo* Wei Hut Xunong Yi, Haiying Liu, and Jing Zhu \\ Laboratory of Light Transmission Optics, \\ South China Normal University, Guangzhou 510630, China
}

(Dated: May 9, 2018)

\begin{abstract}
It is found that the amphoteric refraction, i.e. the refraction can be either positive or negative depending on the incident angles, could occur at a planar interface associated with a uniaxially anisotropic positive index media (PIM) or an anisotropic negative index media (NIM). Particularly, the anomalous negative refraction can occur at a planar interface from an isotropic PIM to an anisotropic PIM, whereas the anomalous positive refraction occurs at the interface from an isotropic PIM to an anisotropic NIM. The optimal conditions to yield the two unusual refractions are obtained. The difference of the two types of amphoteric refraction is discussed.
\end{abstract}

PACS numbers: 78.20.Ci, 41.20.Jb, 42.25.Gy

${ }^{*}$ Author to whom correspondence should be addressed. E-mail: hailuluo@163.com

${ }^{\dagger}$ Author to whom correspondence should be addressed. E-mail: huwei@scnu.edu.cn 


\section{INTRODUCTION}

The material with negative index of refraction, also known as left-handed media, first introduced by Veselago in 1968 [1], has recently received much more attention in literature. Experimentally, an artificial composite media, meta-material, which has negative index of refraction in the microwave regime, has been built and measured [2, 3]. Theoretically, the perfect lens, which can realize sub-wavelength focusing, was first proposed by Pendry [4]. Although some arguments have been evoked on the concepts of negative refractive index and the perfect lens both [5, 6, 7, 8], the phenomenon of negative refraction has been examined and confirmed by many researchers [9, 10, 11, 12, 13, 14, 15].

Negative refraction, in which the tangential component of the Poynting vector changes sign when light refracted, is one of the dramatic properties of the left-handed media. It occurs at the interface between a normal positive index media (PIM), where the electric permittivity $\epsilon$ and magnetic permeability $\mu$ are both positive, and a negative index media (NIM), where $\epsilon$ and $\mu$ are both negative 1]. It was also found recently that negative refraction can occur in some other media. Negative refraction in photonic crystal without negative effective index of refraction has been reported [16, 17]. Lindell et al [18] have shown that negative refraction can occur at an interface associated with a uniaxially anisotropic media, where only one of the four parameters of $\epsilon$ and $\mu$ tensors are negative. Recently, Zhang et al 19] have demonstrated that an amphoteric refraction, i.e. the refraction can be either positive or negative depending on the incident angles, may prevail at the interface between two anisotropic PIM when their optical axes are in different directions. Further, Liu et al 20] have pointed out that the amphoteric refraction, as well as the omnidirectional total transmission, can occur at a planar interfaces associated with a uniaxial media.

Here we investigate the amphoteric refraction characteristics of extraordinary light at the planar interface associated with an anisotropic positive index media or an anisotropic negative index media. It is found that the anomalous negative refraction can occur at a planar interface from an isotropic PIM to an anisotropic PIM, while the anomalous positive refraction occurs at the interface from an isotropic PIM to an anisotropic NIM. Relying on dispersion equation, the optimal optical axis angle and the strongest anomalous refraction angle are obtained. It is noted that for a strong anisotropy media, the maximum incident angle yields the anomalous refraction could be very large. The difference of amphoteric refraction associated with anisotropic PIM and with anisotropic NIM is also discussed. 


\section{AMPHOTERIC REFRACTION OF LIGHT}

The negative refraction at an interface associated with uniaxially anisotropic media, where some of the four parameters of $\epsilon$ and $\mu$ tensors are negative, has been generally discussed [18, 21, 22]. However, in those literature, the optical axis of uniaxial NIM was assumed to be normal or parallel to the interface. Here we assume that there is an angle $\varphi$ between the optical axis and the planar interface. It is the necessary condition for the amphoteric refraction.

We consider the propagation of a planar wave of frequency $\omega$ as $\mathbf{E}=\mathbf{E}_{0} e^{i \mathbf{k} \cdot \mathbf{r}-i \omega t}$ and $\mathbf{H}=\mathbf{H}_{0} e^{i \mathbf{k} \cdot \mathbf{r}-i \omega t}$, through an isotropic PIM toward an uniaxially anisotropic media. For the nonmagnetic isotropic PIM, the relative permittivity and permeability are both scalars, i.e. $\epsilon_{I}$ and $\mu_{I}$. For the convenience and without loss of generality, we assume that the permeability of uniaxially anisotropic media is isotropic, whereas the relative permittivity is a second-rank tensor, given in principal coordinates as

$$
\epsilon=\left(\begin{array}{ccc}
\epsilon_{\perp} & 0 & 0 \\
0 & \epsilon_{\perp} & 0 \\
0 & 0 & \epsilon_{\|}
\end{array}\right) .
$$

We assume that $\epsilon_{\perp}, \epsilon_{\|}>0$ and $\mu>0$ for the anisotropic PIM, and $\epsilon_{\perp}, \epsilon_{\|}<0$ and $\mu<0$ for the anisotropic NIM, in which both ordinary and extraordinary indices are negative. As shown in Fig.1, we also assume that all the wave vectors and the optical axis are in the $x-z$ planar, where the interface is the $x-y$ plane at $z=0$. The isotropic media is on the left hand side of interface, while the anisotropic media on the right hand side. We refer to the angle of the optical axis, $\varphi$, as the angle between the optical axis of anisotropic media and the $x$ direction.

When dealing with an extraordinary plane wave, polarized along $\mathrm{x}$ direction, one have $E_{y}=H_{x}=H_{z}=0$. The iso-frequency surface of the wave vector $\mathbf{k}_{t}$ for transmitted wave is an ellipse described by the dispersion relation. It is worth pointing out that for an extraordinary wave the time-averaged Poynting vector for the transmitted wave $\mathbf{S}_{t}$, which defines the actual direction of the energy flow of the extraordinary light, is normal to the tangential surface of the wave vector ellipse at point $\mathbf{k}_{t}$.

The dispersion relation for extraordinary wave in uniaxial media can be easily obtained by solving Maxwell's equations for plane waves in the principal coordinates[23] with a rotation 

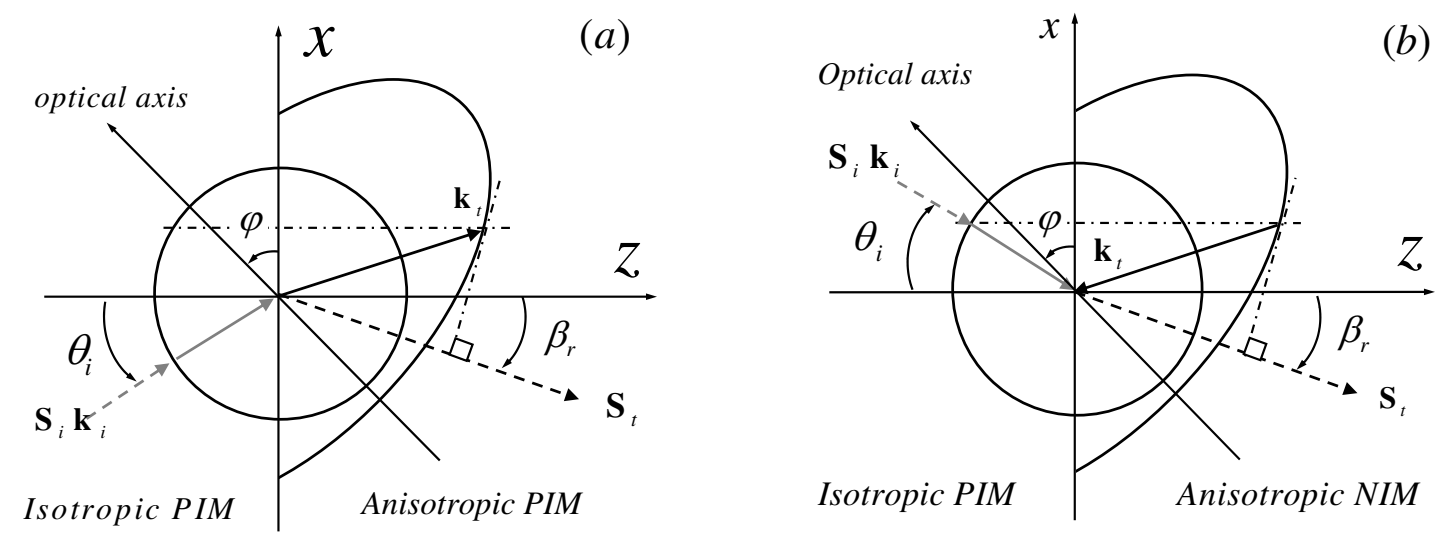

FIG. 1: The mechanisms for the amphoteric refractions. (a) The negative refraction from an isotropic PIM to an anisotropic PIM. (b) The positive refraction from an isotropic PIM to an anisotropic NIM. The iso-frequency wave-vector surfaces for the isotropic media (left) and the anisotropic media (right) are circle and ellipse, respectively. The incident and transmitted wave vectors $\mathbf{k}_{i}$ and $\mathbf{k}_{t}$ are indicated by the solid arrow, whereas the energy flows $\mathbf{S}_{i}$ and $\mathbf{S}_{t}$ are indicated by dashed arrows. The $k_{x}$ components should be continuous across the interface, and the vector $\mathbf{S}_{t}$ is normal to the tangential surface of the iso-frequency wave-vector ellipse at the point $\mathbf{k}_{t}$.

of coordinates on $y$-axis, as

$$
\alpha k_{t x}^{2}+\beta k_{t z}^{2}+\gamma k_{t x} k_{t z}=\frac{\omega^{2}}{c^{2}}
$$

where $k_{t x}$ and $k_{t z}$ represent the $x$ and $z$ components of transmitted wave vector $\mathbf{k}_{t}$ and $\alpha, \beta$ and $\gamma$ are given by

$$
\begin{aligned}
\alpha & =\frac{1}{\epsilon_{\perp} \epsilon_{\|} \mu}\left(\epsilon_{\perp} \sin ^{2} \varphi+\epsilon_{\|} \cos ^{2} \varphi\right) \\
\beta & =\frac{1}{\epsilon_{\perp} \epsilon_{\|} \mu}\left(\epsilon_{\perp} \cos ^{2} \varphi+\epsilon_{\|} \sin ^{2} \varphi\right) \\
\gamma & =\frac{1}{\epsilon_{\perp} \epsilon_{\|} \mu}\left(\epsilon_{\perp} \sin 2 \varphi-\epsilon_{\|} \sin 2 \varphi\right) .
\end{aligned}
$$

The $z$-compnent of the wave vector can be found by the solution of Eq.(2), i.e.

$$
k_{t z}=\frac{1}{2 \beta}\left[\sigma \sqrt{(\gamma-4 \alpha \beta) k_{t x}^{2}+4 \beta \frac{\omega^{2}}{c^{2}}}-\gamma^{2} k_{t x}\right] .
$$

Here $\sigma=1$ for PIM and $\sigma=-1$ for NIM . This choice of sign ensures that light power propagates away from the surface to the $+z$ direction. 
We now determine the angle of refraction. The incident angle of light in the isotropic PIM is $\theta_{i}=\tan ^{-1}\left(k_{i x} / k_{i z}\right)$, and the refractive angle of the transmitted wave vector in anisotropic media can be found by $\theta_{r}=\tan ^{-1}\left(k_{t x} / k_{t z}\right)$. From the boundary conditions at the interface $z=0$, the tangential components of the wave vectors must be continuous, i.e. $k_{t x}=k_{i x}$. Based on Eq.(3) and the boundary conditions, one can obtain

$$
\theta_{r}=\tan ^{-1}\left[\frac{2 \beta \sqrt{\epsilon_{I}} \sin \theta_{i}}{\gamma \sqrt{\epsilon_{I}} \sin \theta_{i}-\sigma \sqrt{\left(\gamma^{2}-4 \alpha \beta\right) \epsilon_{I} \sin ^{2} \theta_{i}+4 \beta}}\right]
$$

It is well know that the actual propagation direction of an extraordinary light in an anisotropic media differs from the direction of its wave vector. In the following of this paper, the direction of an extraordinary light is defined based on the time-averaged Poynting vector $\mathbf{S}=\frac{1}{2} \mathbf{R e}\left(\mathbf{E}^{*} \times \mathbf{H}\right)$, named as ray refractive angle. This ray refractive angle is given by

$$
\beta_{r}=\tan ^{-1}\left(\frac{S_{t x}}{S_{t z}}\right)
$$

For monochromatic extraordinary wave, it can be simplified as $\beta_{r}=\tan ^{-1}\left(-E_{t z} / E_{t x}\right)$. The $x$ and $z$ components of the electric field $\mathbf{E}_{t}$ for transmitted wave can be derived from the equation $\nabla \cdot \mathbf{D}=0$ associated with a rotation of coordinates, i.e.

$$
\begin{array}{r}
\epsilon_{\perp}\left(k_{t x} \cos \varphi-k_{t z} \sin \varphi\right)\left(E_{t x} \cos \varphi-E_{t z} \sin \varphi\right) \\
+\epsilon_{\|}\left(k_{t x} \sin \varphi+k_{t z} \cos \varphi\right)\left(E_{t x} \sin \varphi+E_{t z} \cos \varphi\right)=0 .
\end{array}
$$

Based on Eq.(3), (5), (6), and the boundary continuous conditions, one can derive the equation for the ray refractive angle of extraordinary light,

$$
\beta_{r}=\tan ^{-1}\left[\frac{\left(4 \alpha \beta-\gamma^{2}\right) \sqrt{\epsilon_{I}} \sin \theta_{i}+\sigma \gamma \sqrt{\left(\gamma^{2}-4 \alpha \beta\right) \epsilon_{I} \sin ^{2} \theta_{i}+4 \beta}}{2 \sigma \beta \sqrt{\left(\gamma^{2}-4 \alpha \beta\right) \epsilon_{I} \sin ^{2} \theta_{i}+4 \beta}}\right] .
$$

Due to the uniaxial anisotropy, the time-averaged Poynting vector $\mathbf{S}_{t}$ and the wave vector $\mathbf{k}_{t}$ are in different directions. There is a bending angle between them. Because of the bending angle, for some incident wave the negative refraction can occurs in anisotropic PIM, while the positive refraction can occurs in anisotropic NIM.

At the interface, there are two conditions that the light refraction has to obey. The first is the continuous conditions of the tangential components of the wave vectors, i.e. $k_{t x}=k_{i x}$. The second is the energy conservation condition which requests the normal components $S_{t z}$ and $S_{i z}$ must have the same sign. For the anisotropic PIM shown in Fig.1a, the normal components $S_{t z}$ and $k_{t z}$ are always in the same sign, so we know that $k_{t z}$ and $k_{i z}$ have the 
same sign too. It yields that the wave vector refraction is always positive. In this case, if the tangential components $S_{t x}$ and $k_{t x}$ are in the opposite signs due to the bending angle, it leads to a manifestation of the negative refraction. It is noted that the negative refraction only occurs in a narrow range of incident angles.

It is contrary in the anisotropic NIM as shown in Fig.1b. The normal components $S_{t z}$ and $k_{t z}$ are in the opposite signs now, then $k_{t z}$ and $k_{i z}$ have the different signs. So the wave vector refraction is always negative. The positive refraction also occurs when $S_{t x}$ and $k_{t x}$ have the same sign due to the bending angle. Therefore, the amphoteric refractions are determined by whether the signs of $S_{t x}$ and $k_{t x}$ are same or opposite. Their physical essential is due to the uniaxial anisotropic. For the isotropic media, the Poynting vector $\mathbf{S}_{t}$ is always parallel or anti-parallel with the wave vector $\mathbf{k}_{t}$ in PIM or NIM, respectively. The signs of tangential components $S_{t x}$ and $k_{t x}$ are always same (positive refraction for PIM) or opposite (negative refraction for NIM). There is no amphoteric refraction for isotropic media.

\section{THE REALIZATION OF ANOMALOUS REFRACTIONS}

As an example of amphoteric refraction in anisotropic PIM, the numerical results calculated for a $\mathrm{YVO}_{4}$ crystal, which has recently been proposed by Zhang et al in their experiment [19], is shown in Fig.2a. The $Y V O_{4}$ crystal is a nonmagnetic uniaxial crystal with $\epsilon_{\perp}=n_{0}^{2}=4.07103, \epsilon_{\|}=n_{e}^{2}=5.06614$ and $\mu=1$. For comparison, an anisotropic NIM is shown in Fig.2b, with parameters of $\epsilon_{\perp}=-4.07103, \epsilon_{\|}=-5.06614$ and $\mu=-1$.

In Fig.2, the ray refractive angle $\beta_{r}$ and the wave-vector refractive angle $\theta_{r}$ are shown as the solid and dashed curves, respectively. The negative refraction occurs when curves fall in the second or fourth quadrant of the $\theta_{i}-\beta_{r}$ plane. One can see in Fig.2a that it is always positive refraction for the wave-vector refractive angle $\theta_{r}$. Whereas for the ray refraction angle $\beta_{r}$, the incident angle can be divided into three regions: one negative refraction $\left(\theta_{i} / \beta_{r}<0\right)$ and two positive refraction $\left(\theta_{i} / \beta_{r}>0\right)$. The negative refraction occurs in the fourth quadrant of the $\theta_{i}-\beta_{r}$ plane, i.e. $0 \leq \theta_{i} \leq \theta_{i c}$. From Fig.2b, it is contrary for anisotropic NIM. It is always negative refraction for the wave-vector refractive angle $\theta_{r}$ whereas positive refraction occurs in the third quadrant for $\theta_{i c} \leq \theta_{i} \leq 0$.

It is noted that the negative refraction occurs in the fourth quadrant for a $Y \mathrm{VO}_{4}$ crystal because it is a positive uniaxial crystal, i.e. $\epsilon_{\perp}<\epsilon_{\|}$. Negative refraction should occurs in the second quadrant for a negative uniaxial crystal. However, The conditions for yielding 

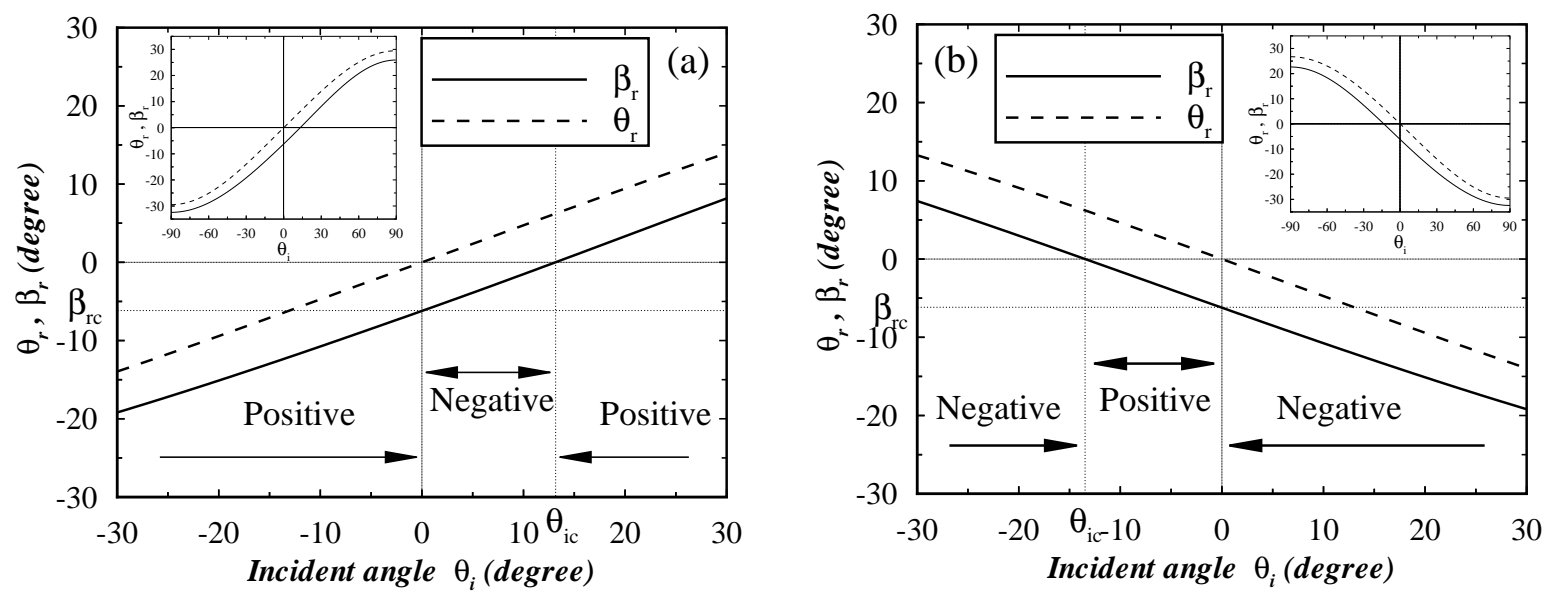

FIG. 2: The variations of the ray refractive angle $\beta_{r}$ for energy flow and the transmitted wave vector angle $\theta_{r}$ as a function of the incident angle $\theta_{i}$, where $\varphi=\pi / 4$. (a) In the anisotropic PIM, the anomalous negative refraction $\left(\theta_{i} / \beta_{r}<0\right)$ occurs in region $0 \leq \theta_{i} \leq \theta_{i c}$. (b) In the anisotropic NIM, the anomalous positive refraction $\left(\theta_{i} / \beta_{r}>0\right)$ occurs in region $0 \geq \theta_{i} \geq \theta_{i c}$. The inset figures are same as main frames.
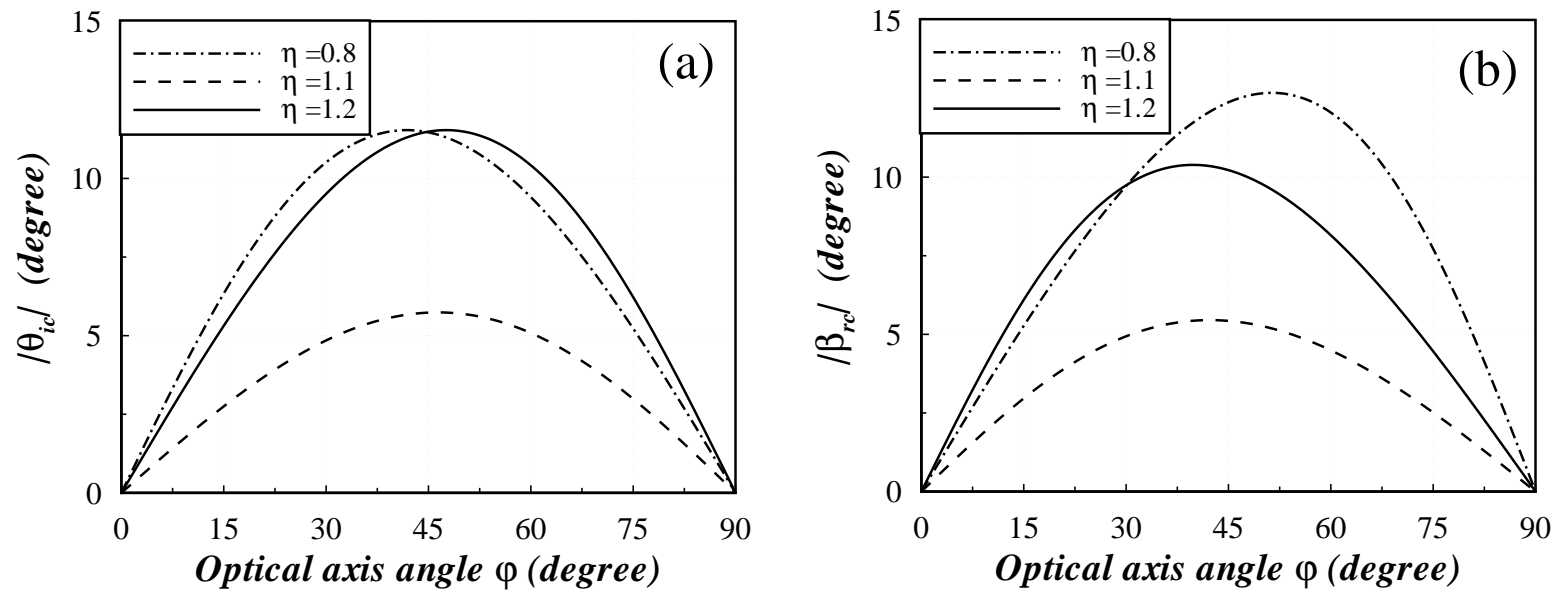

FIG. 3: (a) The dependence of the particular angle $\left|\theta_{i c}\right|$ on the optical axis angle $\varphi$ for several typical anisotropic media. (b) The dependence of the maximum bending angle $\left|\beta_{r c}\right|$ on the optical axis angle $\varphi$ for several typical anisotropic media, where $\eta=\sqrt{\epsilon_{\|} / \epsilon_{\perp}}$ is the anisotropy parameter.

anomalous refractions, which refer to negative refraction in an anisotropic PIM and positive refraction in an anisotropic NIM in following, can be written uniformly as

$$
0 \leq\left|\theta_{i}\right| \leq\left|\theta_{i c}\right|
$$


where $\theta_{i c}$ is the particular incident angle when the ray refractive angle $\beta_{r}=0$. For both anisotropic PIM and anisotropic NIM, $\theta_{i c}$ can be solved from Eq.(5) under the condition $S_{t x}=0$, i.e.

$$
\theta_{i c}=\sin ^{-1}\left[\frac{\sigma\left(\epsilon_{\|}-\epsilon_{\perp}\right) \mu \sin \varphi \cos \varphi}{\sqrt{\epsilon_{I}\left(\epsilon_{\perp} \mu \sin ^{2} \varphi+\epsilon_{\|} \mu \cos ^{2} \varphi\right)}}\right]
$$

The particular incident angle $\theta_{i c}$ is dependent on the optical axis angle $\varphi$, as well as the permittivities $\epsilon_{\perp}$ and $\epsilon_{\|}$, and the permeability $\mu$. Under the isotropic limit $\epsilon_{\perp}=\epsilon_{\|}$, one can obtain $\theta_{i c}=0$. It recovers the well-known fact that no negative refraction can be observed at the interfaces between two isotopic PIMs, and no positive refraction between an isotropic PIM and an isotropic NIM. The dependence of the particular incident angle $\left|\theta_{i c}\right|$ on the optical axis angle $\varphi$ for several typical uniaxial crystal are shown in Fig.3(a). It can be seen when $\varphi=0^{\circ}$ or $90^{\circ}$, the particular incident angle $\theta_{i c}$ approaches to zero, which means there are no amphoteric refraction to be observed. For most anisotropic media, when $\varphi$ is about $45^{\circ},\left|\theta_{i c}\right|$ has its maximum value. To obtain the maximum of the particular incident angle $\theta_{i c}$, the optical axis angle $\varphi$ should be chosen as

$$
\varphi_{o p t}=\cos ^{-1}\left[\sqrt{\frac{1}{1+\eta}}\right] .
$$

And the maximum incident angle $\theta_{i c}^{\max }$ is

$$
\theta_{i c}^{\max }=\sin ^{-1}\left[\frac{\sigma \sqrt{\epsilon_{\perp} \mu}}{\sqrt{\epsilon_{I}}}(\eta-1)\right],
$$

where $\eta=\sqrt{\epsilon_{\|} / \epsilon_{\perp}}$ is the anisotropy parameter. The variations of the optimal optical axis angle $\varphi_{\text {opt }}$ and the maximum incident angle $\theta_{i c}^{\max }$ dependent on the anisotropy parameter $\eta$ are shown in Fig. 4, where we choose $\epsilon_{I}=\epsilon_{\perp} \mu$. As an example by choosing $Y V O_{4}$ crystal, we can obtain the optimal optical axis angle of $\varphi_{\text {opt }}=46.57^{\circ}$ and the corresponding maximum incident angle of $\theta_{i c}^{\max }=13.48^{\circ}$.

We can see that for a strongly anisotropy media, the maximum incident angle could be very large. If there were an anisotropic media satisfied with the condition $\left(\sqrt{\epsilon_{\|} \mu}-\sqrt{\epsilon_{\perp} \mu}\right)=$ $\sqrt{\epsilon_{I}}$, the maximum incident angle could be $\left|\theta_{i c}^{\max }\right|=90^{\circ}$. When $\left(\sqrt{\epsilon_{\|} \mu}-\sqrt{\epsilon_{\perp} \mu}\right)>\sqrt{\epsilon_{I}}$, the Eq. (11) is invalid and one still has $\left|\theta_{i c}^{\max }\right|=90^{\circ}$. In our knowledge, no existing crystal can satisfy this condition. But we confide some artificial anisotropy media could be constructed by using new technique of liquid crystal, photonic crystal, etc, if it is worth in practice. 


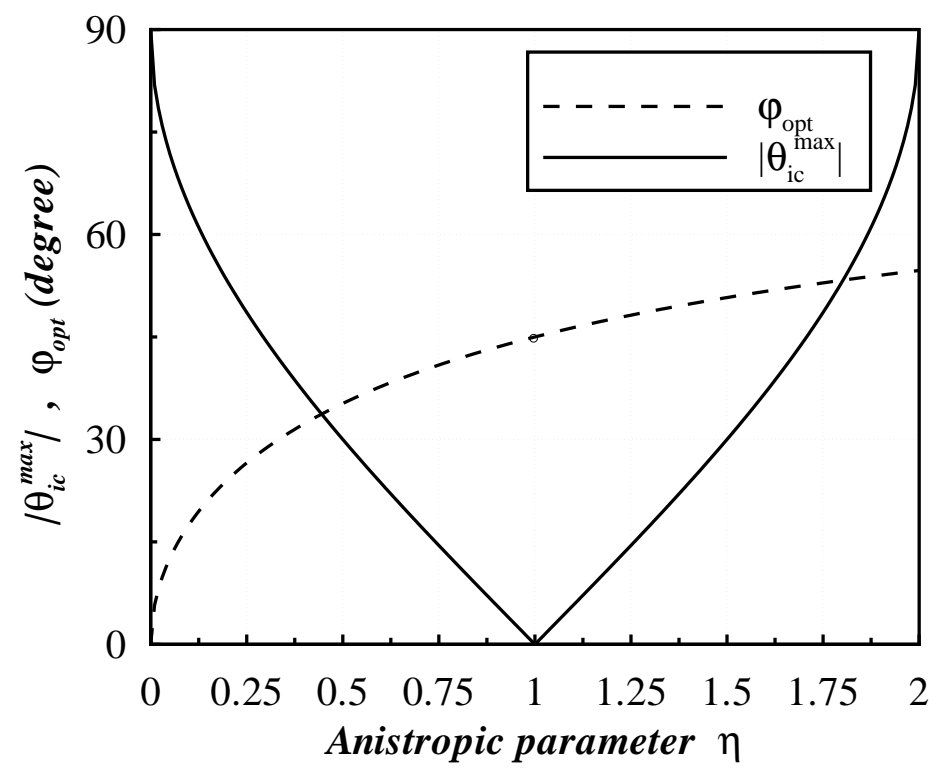

FIG. 4: The optimal optical axis angle $\varphi_{\text {opt }}$ and the maximum incident angle $\left|\theta_{i c}^{\max }\right|$ as a function of the anisotropy parameter $\eta$, where $\sqrt{\epsilon_{I}}=\sqrt{\epsilon_{\perp} \mu}$.

\section{THE STRONGEST ANOMALOUS REFRACTION}

It is also worth noting the particular refractive angle $\beta_{r c}$, which is the refraction angle of light when the incident angle is zero or $k_{t x}=0$. It represents the strongest negative refraction for anisotropic PIM or the strongest positive refraction for anisotropic NIM. This angle is also the particular incident angle of anomalous refraction when the light propagates from an anisotropic media toward an isotropic media. The phenomena of anomalous refraction at the interface from an isotropic NIM toward an anisotropic PIM or PIM and its analysis are similar. So does the anomalous refraction from an anisotropic media toward an isotropic media.

The particular refractive angle $\beta_{r c}$ can be solved from Eq.(5) under the condition $k_{t x}=0$, i.e.

$$
\beta_{r c}=\tan ^{-1}\left[\frac{\left(\eta^{2}-1\right) \sin \varphi \cos \varphi}{\cos ^{2} \varphi+\eta^{2} \sin ^{2} \varphi}\right] .
$$

It is also displayed as a function of the anisotropy parameter $\eta$ and the optical axis angle $\varphi$. The dependence of the particular refractive angle $\left|\beta_{r c}\right|$ on the optical axis angle $\varphi$ for several typical anisotropic media is shown in Fig.3(b). The variations of particular refractive angle $\beta_{r c}$ are similar to that of the particular incident angle $\theta_{i c}$. To obtain the maximum of the 


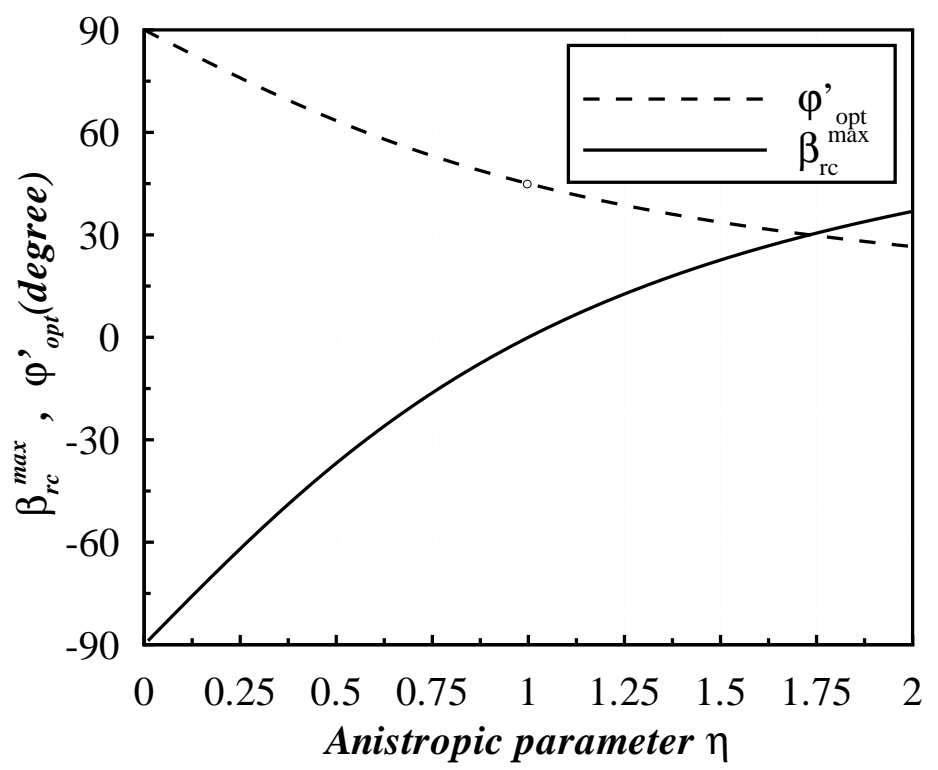

FIG. 5: The optimal optical axis angle $\varphi_{o p t}^{\prime}$ and the maximum bending angle $\beta_{r c}^{\text {max }}$ as a function of the anisotropy parameter $\eta$.

particular refractive angle $\beta_{r c}$, the optical axis angle $\varphi$ should be chosen as

$$
\varphi_{o p t}^{\prime}=\cos ^{-1}\left[\sqrt{\frac{1}{\eta^{2}+1}}\right],
$$

and the maximum bending angle $\beta_{r c}^{\max }$ is

$$
\beta_{r c}^{\max }=\tan ^{-1}\left[\frac{\left(\eta^{2}-1\right)}{2 \eta}\right] .
$$

Unlike the maximum incident angle $\theta_{i c}^{\max }$, the max bending angle $\beta_{r c}^{\max }$ is only dependent on anisotropy parameter $\eta$ but independent on $\epsilon_{I}$. The variations of the optimal optical axis angle $\varphi_{\text {opt }}^{\prime}$ and the maximum bending angle $\beta_{r c}^{\text {max }}$ dependent on the anisotropy parameter $\eta$ are shown in Fig.5. Under the isotropic limit $\eta=1, \beta_{r c}^{\max }=0$, which recovers the fact that no negative refraction can be observed at the interfaces between two isotopic PIMs, and no positive refraction between an isotropic PIM and an isotropic NIM.

\section{DISCUSSIONS AND CONCLUSIONS}

Theoretical investigation above indicates that the amphoteric refraction can be observed at a planar interface between an isotropic media and an anisotropic media. When certain conditions are satisfied, the interface between an isotropic PIM and an anisotropic PIM 
(or NIM) can exhibit negative (or positive) refraction. It is worth noting the anomalous refraction phenomenon occurs only in a narrow range of incident angles for an anisotropic PIM. Even for a very strongly anisotropy parameter, i.e. $\left(\sqrt{\epsilon_{\|} \mu}-\sqrt{\epsilon_{\perp} \mu}\right)=\sqrt{\epsilon_{I}}$, the incident angle is limited in the half planer, i.e. $0^{\circ}<\theta_{i}<90^{\circ}$ or $0^{\circ}>\theta_{i}>90^{\circ}$.

An intriguing phenomenon associated with the negative refraction that has generated considerable interest is partial focusing by an anisotropic NIM planar slab 24, 25]. With the suitable arrangement of the optical axis and the appropriate selection of anisotropy parameter of the anisotropic NIM, the optimal focusing for anisotropic NIM as lens structures can be effectively obtained. Obviously the anomalous positive refraction may impair or weaken the focusing property. We do not expect that the negative refraction in an anisotropic PIM may obtain the same results. However, we believe that some potential applications can be derived based on the amphoteric refractive properties in anisotropic PIM or NIM. They can, for example, be used to provide bending, angular dispersion, energy filtering, and beam collimating for optical devices.

\section{Acknowledgments}

The authors are sincerely grateful to Prof. Qi Guo and L.B. Hu for many fruitful discussions. This work is partially supported by projects of the National Natural Science Foundation of China (No. 60278013), the Team Project of Natural Science Foundation of Guangdong Province (No. 20003061), the Foundation of National Hi-Tech Committee, the Fok Yin Tung High Education Foundation of the Ministry of Education of China (No. 81058).

[1] V.G. Veselago, Sov. Phys. Usp. 10, 509(1968).

[2] D. R. Smith , W. J. Padilla, D. C. Vier, S. C. Nemat-Nasser and S. Schultz, Phys. Rev. Lett. 84, 4184(2000).

[3] R. A. Shelby, D. R. Smith, and S. Schultz, Science 292, 77 (2001).

[4] J. B. Pendry, Phys. Rev. Lett. 85, 3966 (2000).

[5] P. M. Valanju, R. M. Walser, and A. P. Valanju Phys. Rev. Lett. 88, 187401 (2002). 
[6] G.W. Hooft, Phys. Rev. Lett. 87, 249701(2001); J. B. Pendry, Phys. Rev. Lett. 87 249702(2001);

[7] J. M. Williams, Phys. Rev. Lett. 87, 249703(2001); J. B. Pendry, Phys. Rev. Lett. 87249704(2001)

[8] N. Garcia and M. Nieto-Vesperinas Phys. Rev. Lett. 88 207403(2002)

[9] C.G. Parazzoli, R. G.Greegpr, K. Li, B. E. C. Koltenba, and M. Tanielian,Phys. Rev. Lett. 90, 107401 (2003).

[10] D. R. Smith, D. Schurig and J. B. Pendry, Appl. Phys. Lett. 81, 2713 (2002).

[11] J. Pacheco, Jr, T. M. Grzegorczyk, B. I. Wu, Y. Zhang and J. A. Kong, Phys. Rev. Lett. 89, $257401(2002)$.

[12] S. Foteinopoulou, E. N. Economou, and C.M. Soukoulis, Phys. Rev. Lett. 90, 107402 (2003)

[13] A. A. Houck, J. B. Brock, and I. L. Chuang, Phys. Rev. Lett. 90, 137401 (2003)

[14] G. Gomez-Santos, Phys. Rev. Lett. 90, 077401(2003).

[15] Michael W. Feise, Peter J. Bevelacqua, and John B. Schneider Phys. Rev. B 66, 035113(2002)

[16] M. Notomi, Phys. Rev. B 62, 10696 (2000).

[17] C. Luo, S. G. Johnson, J. D. Joannopoulos, and J. P. Pendry, Phys. Rev. B 65, 201104 (2002).

[18] I. V. Lindel, S. A. Tretyakov, K. I. Nikoskinen, and S. Ilvonen, Opt. Technol. Lett. 31, $129(2001)$.

[19] Y. Zhang, B. Fluegel, and A. Mascarenhas, Phys. Rev. Lett. 91, 157401(2003).

[20] Z. Liu, Z. Lin, and S. T. Chui, Phys. Rev. B 69, 115402 (2004).

[21] L. B. Hu and S. T. Chui, Phys. Rev. B 66, 085108 (2002).

[22] D. R. Smith and D. Schurig, Phys. Rev. Lett. 90, 077405 (2003).

[23] A. Yariv and P. Yeh, Optical Wave in Crystals, (John Wiely Sons,New York, 1984) Chap.VI.

[24] D. R. Smith, D. Schurig, J. J. Mock, P. Kolinkp and P. Rye , Appl. Phys. Lett. 84, 2244 (2004).

[25] D. R. Smith,P. Kolinkp and D. Schurig, J. Opt. Soc. Am. B 21, 1032 (2004). 